American Journal of Applied Sciences 6 (5): 952-955, 2009

ISSN 1546-9239

(C) 2009 Science Publications

\title{
The Lateral Reaction of a Deformable Inclined Surface on a Wheel with an Elastic Trunk
}

\author{
Sayel M. Fayyad, Suleiman Abu-Ein and Waleed Momani \\ Department of Mechanical Engineering, Faculty of Engineering and Technology, \\ Al-Balqua Applied University, P.O. Box 15008, Amman, Jordan 11134
}

\begin{abstract}
Problem statement: This study is concerned with a very important problem belonging in the effects of deformable inclined surfaces on a wheel with an elastic trunk. These effects can be represented by two effects: A lateral withdrawal of the trunk and lateral shift of the ground. Also this study aims to study these effects and analyze them mathematically to find a mathematical relation describes them. Approach: A free body diagram of the wheel which represented all forces affected is drawn then starting with the lateral component all angles are calculated, then by building a relations between these different angles and forces, the final relation is derived mathematically which describes the total deformation of the trunk. Results: It was found that inclination angle of the wheel with inclined surface has a nonlinear positive relationship with both trunk deformation and the displacement in $\mathrm{x}$-direction. On the other hand the relation between the inclination angles with both rigidity factor of the trunk and ground volumetric factor is a non-linear negative relationship. Conclusion/Recommendations: The inclination angle was decreasing as both rigidity and ground volumetric factors are increasing. Inclination angle was increasing with the increase in both displacement $(\mathrm{x})$ and trunks' deformation values. The study of trunk deformation was very important since it will lead to reconstruct the wheel system in the future to get more efficient system, and the slipping phenomenon will be easily analyzed in the future.
\end{abstract}

Key words: Lateral withdrawal, lateral shift, corner angle, radial rigidity factor, volumetric factor of ground, free diameter of wheel, elastic trunk.

\section{INTRODUCTION}

The Course movement of a wheel at a cross inclination basic surfaces, characteristic for conditions of work of tractors on slopes, can be defined by both lateral withdrawal of the trunk and lateral shift of a ground. The lateral shift depends on pressure arising in a ground and, hence, the reaction working on a wheel in a lateral direction.

Little works were found in literature that studied such problems. Genya et al. ${ }^{[1]}$ described a steering trajectory analysis for planetary exploration rovers. Analysis of the steering trajectory was an important issue in order to plan and control the motion maneuvers of a planetary rover. Since a rover might easily slip and skid on loose soil, it is difficult to estimate and analyze the motion of the rover or the behavior of its wheels. The authors developed a model that respects the dynamics of each wheel's slip and skid behavior in order to deal with the steering trajectory of the rover. The developed model is called All-Wheel Dynamics
Model. In the all-wheel dynamics model, the behavior of each wheel on loose soil was modeled based on terramechanics. The steering trajectory of the rover was obtained by a numerical simulation using the wheelandvehicle dynamics model. The proposed model was able to correspond to the experimental results with reasonable precision. IP Services ${ }^{[4]}$, announced about a Load sharing wheel assembly for track laying vehicle, this invention related generally to track laying vehicles and more particularly to a load sharing wheel assembly for use in such vehicles.

Track laying vehicles to which the invention can apply may comprise a vehicle body having a longitudinal axis extending between forward and rearward ends thereof and a pair of track assemblies disposed on opposite sides of the vehicle body. Each track assembly may comprise first and second wheels which were spaced apart in the direction of the longitudinal axis with at least one of the wheels defining a drive wheel. the assembly may further included a track which may for example be formed of

Corresponding Author: Sayel M. Fayyad, ${ }^{3}$ Department of Mechanical Engineering, Faculty of Engineering and Technology, Al-Balqua Applied University, P.O. Box 15008, Amman, Jordan 11134 
elastomeric material the track being entrained around the first and second wheels and having a ground engaging surface and an opposite wheel engaging surface with a plurality of power transmitting elements or drive lugs projecting from the wheel engaging surface. The vehicle may further include a plurality of load sharing wheels between the drive wheel and the idler wheel.

\section{MATERIALS AND METHODS}

Governing equations: The lateral component of a vertical loading can be written as:

$\mathrm{R}=\mathrm{G}_{\mathrm{k}} \sin \alpha$

Where:

$\mathrm{G}_{\mathrm{K}}=$ The vertical loading on a wheel

$\alpha=$ The inclination angle or (corner angle) of the lateral load making with the horizontal plane

The corner angle $\alpha$ (corner angle of a cross inclination of a basic surface) is fairly applied in the case of the rigid basis, when the stain of contact of the trunk is in its plane. While in the case of the deformable basis the lateral component of vertical loading working on a wheel is located in a plane, concerning to the surface of contact of the trunk with ground at the centre of pressure of a wheel show in Fig. 1 and 2. Hence, the corner angle $\alpha$ of an inclination of a lateral component is equal to the corner angle of an inclination of this plane.
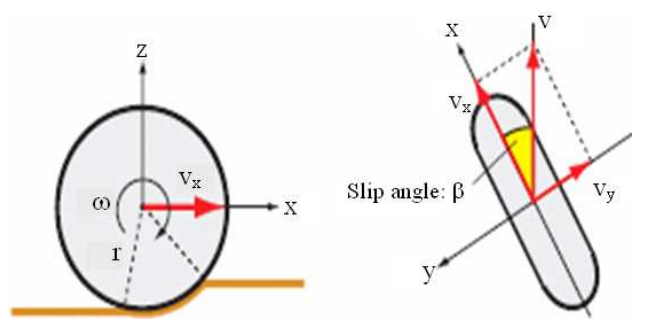

Fig. 1: Contact between the wheel and road ${ }^{[1]}$

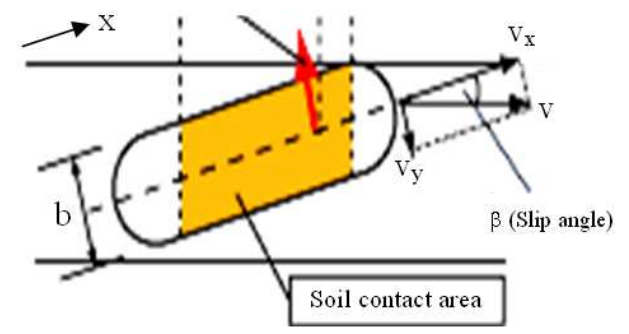

Fig. 2: Stain or contact area ${ }^{[1]}$
To develop a mathematical expression for $\alpha$, were based on an assumption that it is directly proportional to radial deformation of the trunk and return proportionality of the total deformation of the trunk and deformable basis. Depending on such assumption the trunk should be consider as a cylindrical form. In case of its indefinitely large rigidity the corner of an inclination angle $\alpha$ of lateral making vertical loading is equal to a corner of an inclination of a structure of cross section i.e., in this case it will be equal to zero as the deformation of the trunk is equal to zero. Inclination angles have many cases depending on the relationship between the trunk and rigid basis, firstly: This corner angle has a maximum value which is equal to the corner angle of an inclination of a surface of the basis i.e., $\alpha=a$. Secondly if the deformations of the trunk and basis are identical, the corner of an inclination of lateral making vertical loading on a wheel in a stain of contact according to the accepted assumption is equal to half of corner of an inclination of a surface of the basic base:

$\alpha^{\prime}=a / 2$

If the deformation of the trunk is less than twice of the deformation of the base then:

$\alpha^{\prime}=\mathrm{a} / 3$

Otherwise, when the deformation of the base is less than twice the deformation of the trunk:

$\alpha^{\prime}=(2 / 3) \mathrm{a}$

where, $\mathrm{a}$ is the corner angle of an inclination of a surface of the basic base.

The considered special cases have allowed making a general expression for a corner of an inclination angle of lateral making vertical loading on the wheel with a cylindrical racing path:

$\alpha^{\prime}=\mathrm{a} \frac{\mathrm{h}_{\mathrm{m}}}{\mathrm{h}_{\mathrm{m}}+\mathrm{h}_{\mathrm{r}}}$

Where:

$\mathrm{h}_{\text {III }}=$ The maximal radial deformation according to trunk

$\mathrm{h}_{\mathrm{r}}=$ The maximal radial deformations according to the bases in section which is taking place through the centre of pressure of the wheel on a distance from a longitudinal plane of its symmetry

To derive an expression for the corner angle of an inclination of lateral making vertical loading on a wheel 
at any structure of the racing path, meaning, that the considered force is directed on his structure in a point of its appendix (centre of pressure, Fig. 1), so it can be written as:

$$
\alpha^{\prime}=\left(\mathrm{a}-\alpha_{0}\right) \frac{\mathrm{h}_{\mathrm{w}}}{\mathrm{h}_{\mathrm{r}}+\mathrm{h}_{\mathrm{ul}}}+\alpha_{0}
$$

where, $\alpha_{0}$ is the angle of an inclination related to a structure of cross section of a racing path at the centre of pressure of the trunk in the non-loaded condition.

The following equation describing the structure of a racing path, angular factor can be calculated by differentiation. So, the structure of cross section of a racing path of trunks of tractors will be as a square parabola (divergence of ordinates of a parabola precisely enough describes and the real structure is not exceeded by $1 \%$ :

$f(x)=\frac{4 d}{B^{2}} x^{2}$

Where:

$\mathrm{B}=$ The width of a structure

$\mathrm{d}=$ The coordinate of its extreme point (Fig. 1)

From Eq. 7 angular factor can be given as:

$\frac{\mathrm{df}(\mathrm{x})}{\mathrm{dx}}=\frac{8 \mathrm{~d}}{\mathrm{~B}^{2}} \mathrm{x}$

Then $\alpha$ is a function of $\mathrm{X}$ such as:

$\alpha^{\prime}(\mathrm{x})=\arctan \left(\frac{8 \mathrm{~d}}{\mathrm{~B}^{2}} \mathrm{x}\right)$

Corner angle of an inclination related to a structure of cross section of a racing path in a point, the distance with which is equal to displacement of the centre of pressure e, then:

$$
\alpha_{0}^{\prime}=\arctan \left(\frac{8 \mathrm{~d}}{\mathrm{~B}^{2}}\right)
$$

Depending on last equations $\alpha$ can be given as:

$$
\alpha^{\prime}=\left(\mathrm{ah}_{\mathrm{m}}+\alpha_{0} \mathrm{~h}_{\mathrm{r}} / \mathrm{h}_{\mathrm{c}}\right.
$$

where, $h_{c}$ is the deformation of the trunk and basis in cross section which is taking place through the centre pressure of the wheel.

Having replaced $h_{\text {III }}$ and $h_{r}$ by their expressions used $^{[3]}$ in Eq. 11, $\alpha$ can be rewritten as:

$$
\alpha^{\prime}=\frac{a_{0} c+a k \sqrt{D_{0} B B_{\alpha} h_{c}}}{c+k \sqrt{D_{0} B B_{\alpha} h_{c}}}
$$

Where:

$\mathrm{c}=$ The factor of radial rigidity of the trunk

$\mathrm{k}=$ The volumetric factor of a ground given in the sizes of a wheel ${ }^{[5]}$

$D_{0}=$ The free diameter of a wheel

$\mathrm{B}_{\mathrm{a}}=$ Width of a stain of contact

In Eq. 12 the factors most essentially influencing character of interaction of an elastic wheel with the deformable inclined basis are taken into account. On expressions 12 and 1 it is possible to settle an invoice lateral reaction (basic characteristic of this interaction) with the large accuracy. Relations between $\alpha$ and the factor of radial rigidity of trunk, $c$, and the volumetric factor of the ground in the sizes of the wheel, $\mathrm{k}$ and the deformation of the trunk, $h_{c}$ will be represented graphically. In addition the relation between $\alpha$ as a function of $\mathrm{X}$ will be represented graphically.

\section{RESULTS}

The results are demonstrated graphically to show the behavior of the inclination angles and the other main parameters. Equation 12 represents a design relations between the inclination angle and many parameters, these relations are represented graphically as shown in Fig. 3-6. Figure 3 shows the relation between the inclination angle $\left(\alpha^{\prime}\right)$ and the rigidity factor of the trunk, it can be concluded that as the rigidity factor increase inclination angle decrease, in a non-linear behavior. The relation between inclination angle and the trunk deformation is a non-linear positive relation, because as the trunk deformation increase the inclination angle increase as shown in Fig. 4.

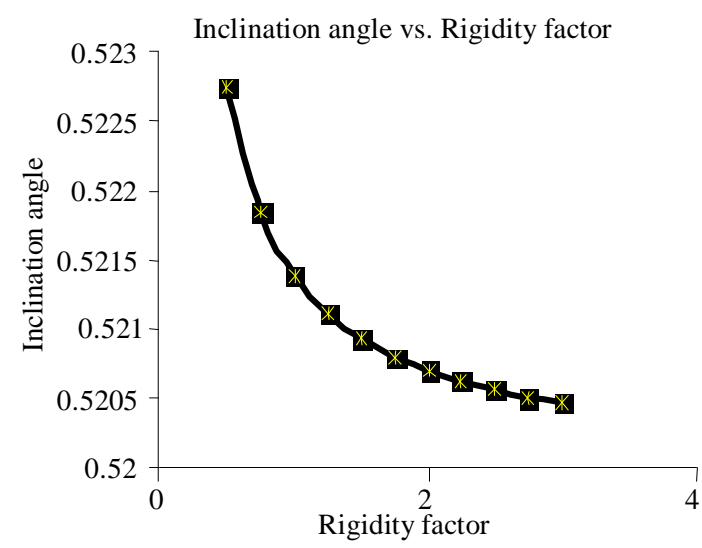

Fig. 3: Inclination angle vs. rigidity factor of the trunk 
Am. J. Applied Sci., 6 (5): 952-955, 2009

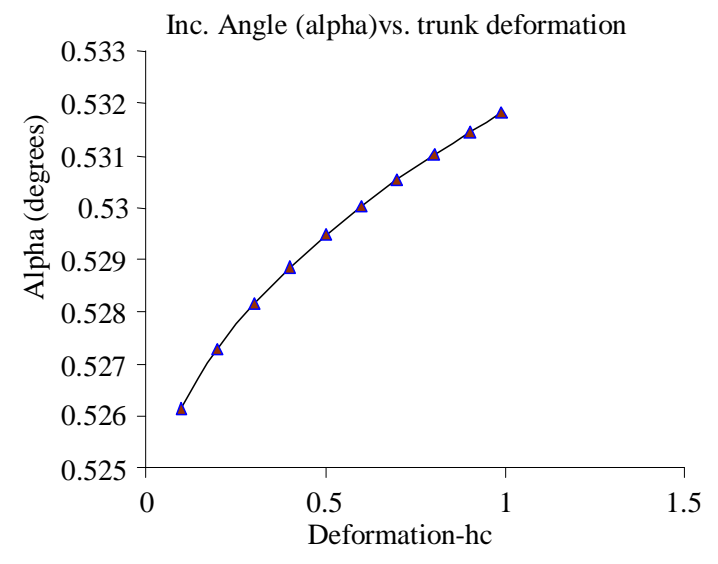

Fig. 4: Inclination angle vs. trunk deformation

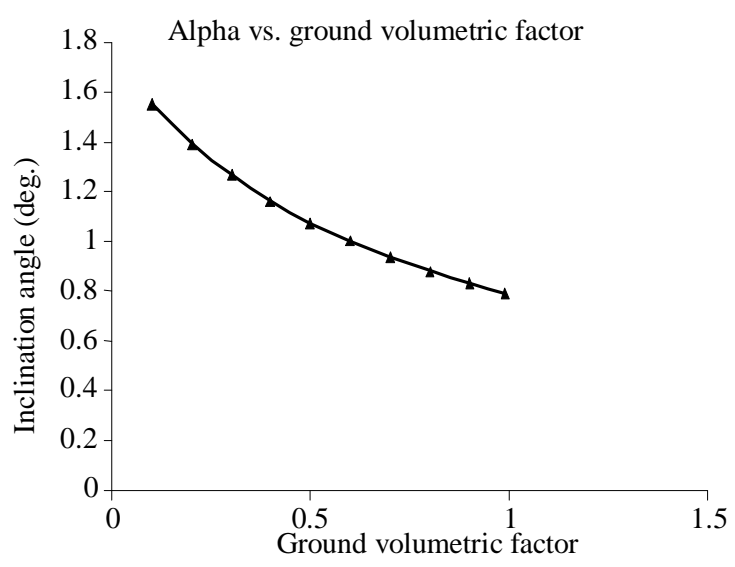

Fig. 5: Inclination angle vs. ground volumetric factor

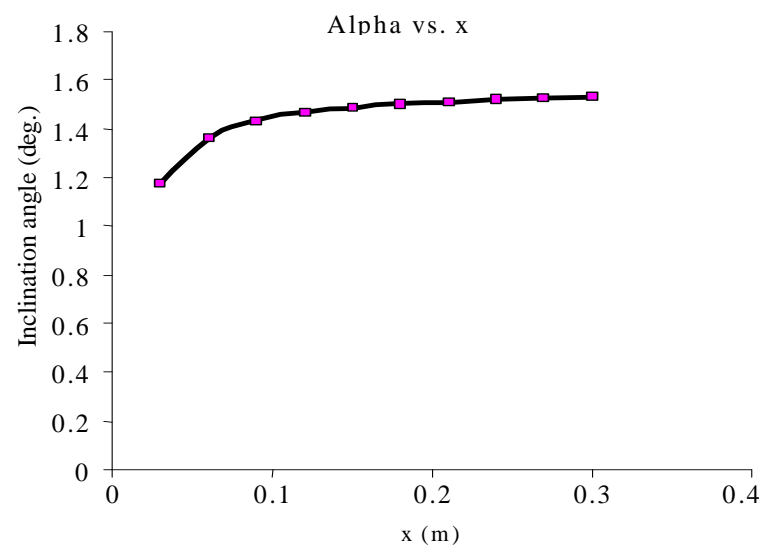

Fig. 6: Inclination angle vs. displacement in x-direction

On the contrary the relation between the inclination angle and ground volumetric factor is non-linear negative relation, as shown in Fig. 5. Also Fig. 6 shows the relation between inclination angle and the displacement in $\mathrm{x}$-direction, it is obvious that they have a non-linear positive relationship. It is difficult to compare the present results with other may available in literatures because they are not available.

\section{DISCUSSION}

Inclination angle has anon-linear negative relation ship with both rigidity and ground volumetric factors the reason beyond that is as the rigidity of the ground becomes higher the stiffness decreases and then the inclined angle decreases. This will forma good base to redesign such systems to give new and good systems which may perform the tasks in more efficient way. Also Alpha increases with deformation, this can be explained such that as deformation increases the surface area increases and so alpha increases.

\section{CONCLUSION}

The following conclusions can be carried out:

- Inclination angle is deceasing as both rigidity and ground volumetric factors are increasing

- Inclination angle is increasing with the increase in both displacement (x) and trunks' deformation values

\section{REFERENCES}

1. Genya, I., M. Akiko and Y. Kazuya, 2001. Steering trajectory analysis of planetary exploration rovers based on all-wheel dynamics model. Proceeding of the 8th International Symposium on Artificial Intelligence, Robotics and Automation in Space, Sept. 5-8, European Space Agency, Munich, Germany, pp: 1-8. http://adsabs.harvard.edu/abs/2005aira.confE..16I.

2. Green, B., 1982. To Derive an Equation of Interaction of a Wheel With a Slope: The Theory and Designing of Mobile Machines. 1st Edn., Minsk University Publications, pp: 63-67.

3. Gyckob, B., 1977. Tractors: The Theory. 1st Edn., Minsk University Publications, pp: 384-400.

4. IP Services, 1998. Load sharing wheel assembly for track laying vehicle http://www.wipo.int/pctdb/en/wo.jsp?wo=1998016 419.

5. Kohobajiob, I., 1972. An Estimation of Lateral Coupling of Wheel Tractors at Work on Slopes. Minsk University Publications, pp: 124-129. 